\title{
Action Function Formulation for Conservative Systems with Second-Order Lagrangian
}

\author{
Ola A. Jarab'ah ${ }^{1}$ \\ ${ }^{1}$ Applied Physics Department, Faculty of Science, Tafila Technical University, Jordan \\ Correspondence: Ola A. Jarab'ah, Applied Physics Department, Faculty of Science, Tafila Technical University, \\ Jordan. E-mail: oasj85@yahoo.com
}

Received: May 11, 2018

Accepted: June 30, 2018

Online Published: July 14, 2018

doi:10.5539/apr.v10n4p50

URL: https://doi.org/10.5539/apr.v10n4p50

\begin{abstract}
The Euler Lagrange equation is studied to obtain the equations of motion for conservative systems with second order Lagrangian. The solutions of these equations are substituted in the given Lagrangian. The action function is then derived by calculating the time integral of the Lagrangian. To explain the application of our formalism two examples are discussed.
\end{abstract}

Keywords: Conservative Systems, Euler Lagrange Equation, Action Function, Generalized Coordinates, Equations of Motion

\section{Introduction}

For conservative systems, there is a famous formulation of classical mechanics known as the Lagrangian formulation (Goldstein, 1980). The Lagrangian function $\mathbf{L}$ for a system is defined to be the difference between the kinetic and potential energies expressed as a function of generalized coordinates $q_{i}(t)$, and velocities $\dot{q}_{i}(t)$. Here the over dot denotes differentiation with respect to time $\mathbf{t}$.

The Lagrangian function $\mathbf{L}$ satisfies the so called Euler-Lagrange equation which is given by:

$$
\frac{d}{d t}\left(\frac{\partial L}{\partial \dot{q}}\right)-\frac{\partial L}{\partial q}=0
$$

Thus, the equations that result from application of equation (1) to a particular Lagrangian are the so called equations of motion.

Although most physical systems can be described by regular Lagrangian that depend at most on the first derivatives of the dynamical variables, the Euler Lagrange equation of a first order Lagrangian is one of the most known and widely used variational equations in mathematics, mechanics and physics.

The study of singular Lagrangian systems was initiated by (Dirac, 1964, 1950). He developed the basic theories of the classical treatment and quantization of such systems. Other researchers (Faddeev, 1988; Dyson, 1990) followed Dirac and showed interest in singular theories. A new formalism for investigating first order singular systems, the canonical was developed by (Rabei et al., 1992). This method was generalized to singular system with higher order Lagrangian (Pimentel et al., 1996, 1998). A treatment of singular Lagrangian system as field system was studied in (Farahat, 1995, 2002, 2006). An alternative method is the Hamilton-Jacobi formulation or the canonical method which is developed by (Guler, 1992; Nawafleh et al., 2004, 2008).

The treatment of theories with higher order derivatives has been first developed by (Ostrogradski, 1850; Pon, 1988) and allows writing the Euler Lagrange equations introduce conjugated momenta and develop Hamilton formalism for such systems.

Systems with higher order Lagrangian have been studied with increasing interest because they appear in many relevant physical problems and in many models in theoretical and mathematical physics, they also appear in some problems of fluid mechanics, electric networks and classical physics. There is a continuing interest in the so called generalized dynamics, that is, the study of physical systems described by Lagrangian containing derivatives of order higher than the first (Muslih, 2002; Nawafleh, 2011). 
Evolution of the action function has been investigated using Hamilton - Jacobi equation (Arnold, 1989; Lanczos, 1986). Recently obtaining the action function for dissipative systems is investigated within the framework of the Hamilton-Jacobi equation, this function is determined using the method of separation of variables (Jarab'ah et al., 2013, 2014). In addition, action function formulation for irregular first order Lagrangian has been studied by (Jarab'ah, 2017). Our aim here is to make a formal generalization of this function for conservative systems with second order Lagrangian in the same manner as for first order Lagrangian.

This paper is organized as follows. In Section 2, the formulation of the action function for conservative systems with second order Lagrangian is discussed. In Section 3, the conservative systems with second order Lagrangian are examined using illustrative examples. Finally, in section 4, the work closes with some concluding remarks.

\section{Determination of the Action Function with Second Order Lagrangian}

The Lagrangian formulation of conservative systems with second-order Lagrangian is given by $L=L(q, \dot{q}, \ddot{q}, t)$

The corresponding Euler Lagrange equations are given by:

$$
\begin{aligned}
& \frac{d^{2}}{d t^{2}}\left(\frac{\partial L}{\partial \ddot{q}_{i}}\right)-\frac{d}{d t}\left(\frac{\partial L}{\partial \dot{q}_{i}}\right)+\frac{\partial L}{\partial q_{i}}=0 \\
& i=1,2,3, \ldots
\end{aligned}
$$

Here we center our attention on the case of a system described by a Lagrangian containing time derivatives of the coordinates up to second order Lagrangian. Using Euler Lagrange equations, we find the equations of motion from the corresponding Lagrangian in terms of the generalized coordinates and their derivatives. Then, we substitute the solutions of the equations of motion in the given Lagrangian. Finally, the integral of the Lagrangian between two instants of time $t_{1}$ and $t_{2}$ is defined as the action function $\mathbf{S}$ which takes this form:

$$
S=\int_{t_{1}}^{t_{2}} L(q, \dot{q}, \ddot{q}, t) d t
$$

Also we can write the accelerations $\ddot{q}_{i}(t)$ as functions of the coordinates $q_{i}(t)$ and velocities $\dot{q}_{i}(t)$.

\section{Examples}

1- This Lagrangian describes the one-dimensional motion of a black box in which a harmonic oscillator is hidden and the angular frequency of oscillations is one (Olga, 1997).

$$
L=\frac{1}{2}\left[\ddot{q}^{2}-\dot{q}^{2}\right]
$$

Using Euler - Lagrange equation

$$
\frac{d^{2}}{d t^{2}}\left(\frac{\partial L}{\partial \ddot{q}}\right)-\frac{d}{d t}\left(\frac{\partial L}{\partial \dot{q}}\right)+\frac{\partial L}{\partial q}=0
$$

Eq. (5) can readily be solved to give

$$
q^{\prime \prime \prime \prime}+q^{\prime \prime}=0
$$

The solution of Eq. (6) is

$$
q(t)=C+D t-A \cos t-B \sin t
$$

Taking the first time derivative of Eq. (7)

$$
\dot{q}=D+A \sin t-B \cos t
$$

By squaring Eq. (8), one gets

$$
\dot{q}^{2}=D^{2}+2 A D \sin t-2 B D \cos t-2 A B \sin t \cos t+A^{2} \sin ^{2} t+B^{2} \cos ^{2} t
$$


Thus, taking the second time derivative of Eq. (7), this gives

$$
\ddot{q}=A \cos t+B \sin t
$$

The square of equation (10) is

$$
\ddot{q}^{2}=A^{2} \cos ^{2} t+B^{2} \sin ^{2} t+2 A B \sin t \cos t
$$

Substituting Eq. (9) and Eq. (11) into Eq. (4), we have

$$
L=\frac{A^{2}}{2} \cos 2 t-\frac{B^{2}}{2} \cos 2 t+A B \sin 2 t-\frac{D^{2}}{2}-A D \sin t+B D \cos t
$$

Making use of Eq. (3)

$$
S=\int_{0}^{t}\left[\frac{A^{2}}{2} \cos 2 t-\frac{B^{2}}{2} \cos 2 t+A B \sin 2 t-\frac{D^{2}}{2}-A D \sin t+B D \cos t\right] d t
$$

Further, the action function takes this form

$$
S=\frac{A^{2}}{4} \sin 2 t-\frac{A B}{2} \cos 2 t+\frac{A B}{2}-\frac{B^{2}}{4} \sin 2 t-\frac{D^{2}}{2} t+A D \cos t-A D+B D \sin t
$$

And the acceleration is

$$
\ddot{q}=A \cos t+B \sin t
$$

2- As a second example, we consider the following regular Lagrangian (Hasan et al., 2004).

$$
L=\frac{1}{2} \ddot{q}^{2}+q \dot{q}
$$

The corresponding Euler Lagrange equation reads

$$
\frac{d^{2}}{d t^{2}}\left(\frac{\partial L}{\partial \ddot{q}}\right)-\frac{d}{d t}\left(\frac{\partial L}{\partial \dot{q}}\right)+\frac{\partial L}{\partial q}=0
$$

Simple manipulations yield

$$
q^{\prime \prime \prime \prime}=0
$$

The solution of Eq. (16) is

$$
q(t)=A+B t+C t^{2}+D t^{3}
$$

Like the previous example. Taking the first time derivative and the second time derivative of Eq. (17), we obtain

$$
\dot{q}=B+2 C t+3 D t^{2}
$$

and

Squaring of Eq. (19), we find that

$$
\ddot{q}=2 C+6 D t
$$

$$
\ddot{q}^{2}=36 D^{2} t^{2}+4 C^{2}+24 C D t
$$

Multiplying Eq. (17) by Eq. (18), this leads to

$$
q \dot{q}=A B+2 C A t+3 D A t^{2}+B^{2} t+3 C B t^{2}+4 D B t^{3}+2 C^{2} t^{3}+5 D C t^{4}+3 D^{2} t^{5}
$$

Putting Eqs. (20) and (21) into Eq. (15), then the Lagrangian can be written as

$$
L=18 D^{2} t^{2}+2 C^{2}+12 C D t+A B+2 C A t+3 D A t^{2}+B^{2} t+3 C B t^{2}+4 D B t^{3}+2 C^{2} t^{3}+5 D C t^{4}+3 D^{2} t^{5}
$$


Using Eq. (3) to find the action function

$$
S=\int_{0}^{t}\left[18 D^{2} t^{2}+2 C^{2}+12 C D t+A B+2 C A t+3 D A t^{2}+B^{2} t+3 C B t^{2}+4 D B t^{3}+2 C^{2} t^{3}+5 D C t^{4}+3 D^{2} t^{5}\right] d t
$$

Then, after integration

$$
S=6 D^{2} t^{3}+2 C^{2} t+6 C D t^{2}+A B t+C A t^{2}+D A t^{3}+\frac{B^{2}}{2} t^{2}+C B t^{3}+D B t^{4}+\frac{C^{2}}{2} t^{4}+C D t^{5}+\frac{D^{2}}{2} t^{6}
$$

and the acceleration is

$$
\ddot{q}=2 C+6 D t
$$

\section{Conclusion}

In this work, we have studied conservative systems with second order Lagrangian to find the action function using Euler Lagrange equations. By substituting the solutions of equations of motion in the given Lagrangian; and by finding the time integral of this Lagrangian we can determine the action function $\mathbf{S}$ as a function of time, also we have determined the acceleration. We illustrated through two examples how the Euler Lagrange equations can be used to find the action function.

\section{References}

Arnold, V. I. (1989). Mathematical Methods of Classical Mechanics (4th ed.). Berlin: Springer-Verlag. https://doi.org/10.1007/978-1-4757-2063-1

Dirac, P. A. M. (1950). Generalized Hamiltonian Dynamics. Canadian Journal of Mathematical Physics, 2, 129148. https://doi.org/10.4153/CJM-1950-012-1

Dirac, P. A. M. (1964). Lectures on Quantum Mechanics. Belfer Graduate School of Science. Yeshiva University, New York.

Dyson, J. F. (1990). Feynman's Proof of the Maxwell's Equations. American Journal of Physics, 58(3), 209-211. https://doi.org/10.1119/1.16188

Faddeev, L., \& Jackiw, R. (1988). Hamiltonian Reduction of Unconstrained and Constrained Systems. Physical Review Letters, 60, 1692. https://doi.org/10.1103/PhysRevLett.60.1692

Farahat, N. I., \& Guler, Y. (1995). Singular Lagrangians as Field Systems. Physical Review A, 51(68) https://doi.org/10.1103/PhysRevA.51.68

Farahat, N. I., \& Heles, M. R. (2006). A Treatment of A Higher-Order Singular Lagrangian as Fields System. Turkish Journal of Physics, 30, 67-79.

Farahat, N. I., \& Ramadan, K. A. (2002). A Treatment of Second -Order Singular Lagrangians as Continuous Systems. Hadronic Journal, 25, 291.

Goldstein, H. (1980). Classical Mechanics (2nd ed.). Addison-Wesley, Reading-Massachusetts.

Guler, Y. (1992). Integration of Singular Systems. Il Nuovo Cimento B, 107(12), 1389-1395.

Hasan, E. H., Rabei, E. M., \& Ghassib, H. B. (2004). Quantization of Higher-Order Constrained Lagrangian Systems Using the WKB Approximation. International Journal of Theoretical Physics, 43(4), 1073-1096. Retrieved from http://link.springer.com/article/10.1023/B:IJTP.0000049027.45011.37

Jarab'ah, O. (2017). The Principal Function for Irregular Lagrangian. Advanced Studies in Theoretical Physics, 11(12), 669-677. https://doi.org/10.12988/astp.2017.71050

Jarab'ah, O., Hasan, E. H., \& Nawafleh, K. (2014). Quantization of Dissipative Systems with Second Order Lagrangian. European Scientific Journal, 10(9), 135-142. Retrieved from https://eujournal.org/index.php/esj/ article/view/3049

Jarab'ah, O., Nawafleh, K., \& Ghassib, H. (2013). A Hamilton - Jacobi Treatment of Dissipative Systems. European Scientific Journal, 9(30), 70-81. Retrieved from http://www.eujournal.org/index.php/esj/article/ download/1946/1888

Lanczos. C. (1986). The Variational Principles of Mechanics. Dovers Publications. 
Muslih, S. I. (2002). Quantization of Singular Systems with Second-Order Lagrangians. Modern Physics Letters A, 17(36), 2383-2391.

Nawafleh, K. I. (2011). Canonical Quantization of Higher Order Lagrangians. Journal of Applied Mathematics. http://doi:10.1155/2011/375838

Nawafleh, K. I., \& Alsoub, A. A. (2008). Quantization of Higher Order Regular Lagrangians as First Order Singular Lagrangians Using Path Integral Approach. Jordan Journal of Physics, 1(2), 73-78.

Nawafleh, K., Rabei, E., \& Ghassib, H. (2004). Hamilton-Jacobi Treatment of Constrained Systems, International Journal of Modern Physics A, 19(3) 347-354. https://doi.org/10.1142/s0217751x04017719

Olga, K. (1997). The Geometry of Ordinary Variational Equations (Lectures Notes in Mathematics 1678). Springer-Verlag, Berlin.

Ostrogradski, M. V. (1850). Mémoires de l'Académie de Saint Petersburg, 6, 385.

Pimentel, B. M., \& Teixeira, R. G. (1996). Hamilton - Jacobi Formulation for Singular Systems with SecondOrder Lagrangians. Il Nuovo Cimento B, 111, 841-854.

Pimentel, B. M., \& Teixeira, R. G. (1998). Generalization of The Hamilton-Jacobi Approach for Higher-Order Singular Systems. Il Nuovo Cimento B, 113. https://www.sif.it/riviste/sif/ncb/econtents/1998/113/06/article/1

Pons, J. M. (1988). Ostrogradski's Theorem for Higher-Order Singular Lagrangians. Letters in Mathematical Physics, 17, 181-189. https://link.springer.com/article/10.1007/BF00401583

Rabei, E., \& Guler, Y. (1992). Hamilton - Jacobi Treatment of Second-Class Constraints. Physical Review A, 46(6), 3513-3515. Retrieved from http://link.aps.org/pdf/101103/physRevA.46.3513

\section{Copyrights}

Copyright for this article is retained by the author(s), with first publication rights granted to the journal.

This is an open-access article distributed under the terms and conditions of the Creative Commons Attribution license (http://creativecommons.org/licenses/by/4.0/). 